\title{
Functional richness: Overview of indices and underlying concepts
}

\author{
Legras G. ${ }^{1,{ }^{*}}$, Loiseau Nicolas ${ }^{1,2}$, Gaertner Jean-Claude ${ }^{3}$
}

${ }^{1}$ Univ Polynesie Francaise, UMR 241, EIO, UPF,IRD,Ifremer,ILM, F-98713 Papeete, French Polynesi, Fr Polynesia.

${ }^{2}$ Univ Montpellier, UMR 9175, MARBEC, IRD,CNRS,IFREMER,UM, F-34095 Montpellier, France.

${ }^{3}$ Ctr IRD Tahiti, ILM Labex Corail, IFREMER, IRD,UMR 241,EIO,UPF, F-98713 Papeete, French

Polynesi, Fr Polynesia.

*Corresponding author : G. Legras, email address : gaelle.legras@upf.pf

\begin{abstract}
:
Functional richness, currently defined as the amount of niche space occupied by the species within a community, is one of the three major components of functional diversity. Different indices have been developed in order to quantify this component. However, the range of indices available for assessing functional richness, often mathematically complex and based on different rationales, can cause confusion for field ecologists and lead to misinterpretation of the results obtained. In this context, we have provided the first study exclusively focused on the comparison of the definitions, advantages and drawbacks of a large set of functional richness indices. The first part of this work is focused on four indices (FDP\&G, FRic, TOP and N-hypervolumes indices) that are currently the most commonly used for assessing functional richness. We have completed our study by including recently developed indices that enable us to take into account the intraspecific trait variability (i.e. FRim index and TDP framework), because there is currently a growing scientific consensus regarding the necessity of including this aspect in the assessment of the functional diversity of communities. We demonstrate that although authors have argued that their index describes the functional richness, each of them describes only part of it, and this part may strongly differ from one index to another. Rather than advocating the general use of a single index and/or systematically avoiding others, our study highlights the need for selecting indices in close relation with the context, the available data and the aims of each study. Such a strategy is an essential preliminary step for preventing misunderstanding and artefactual controversies. Along these lines, we propose some guidelines to help users in selecting the most appropriate indices according both to the facet of functional richness on which they wish to focus and to the characteristics of the available data.
\end{abstract}




\section{Highlights}

- Functional richness is a multi-component concept. Each indices only describes one part of this concept. Index response to disturbances varies according to the index used. Need to use indices in the complementary way. Need to select indices in close relation with the context and the aim of each study.

Keywords : Functional richness indices, Index properties, Limitations, Guidelines 


\section{INTRODUCTION}

Assessing functional diversity, which is now recognized as a main driver of ecosystems functioning and their responses in both terrestrial and aquatic environments, has become a crucial challenge in community ecology. This broad concept is now usually split into three major components: functional richness, functional evenness and functional divergence (Mouillot et al. 2005, Mason et al. 2005, Mouillot et al. 2013). The assessment of functional richness is probably the oldest and the most widely used way of investigating functional diversity (see Diaz and Cabido 2001). However, behind this term, simple in appearance, lies a much more complex component which may widely vary according to the authors and the indices used for its assessment. Initially, functional richness was mainly assessed based on the number of predefined functional groups in a given assemblage. This approach consisted of grouping together all species sharing similar taxonomic, phylogenetic or morphological characteristics (Blondel 2003). It was then assumed that all species belonging to the same functional group have similar effects on ecosystems (Chapin et al. 1996, Petchey \& Gaston 2002, Lavorel \& Garnier 2002). Although some studies have demonstrated that the number of functional groups was highly correlated with the ecosystem productivity (Tilman et al. 1997, Hector et al. 1999, Reich et al. 2001), and might be a good proxy of ecosystem functioning (Petchey et al. 2004), assessing the functional richness through the assessment of pre-defined functional groups can be problematic. The methods used to assign species to each group are in fact highly subjective because they are based on the user's choices (Chapain et al. 1996, Holmes et al. 1979, Diaz \& Cabido 2001). The definition of the boundaries between each group determines the number of functional groups and by consequence, may impact which species are included within each group. If we assume that the number of functional groups is in direct relation with the ecosystem functioning, defining this number in a non-standardized way can produce undesirable variability in the results obtained. Furthermore, the use of functional groups ignores all possible differences between species belonging to a single group, whereas these differences can be important notably when the functional traits are quantitative (Petchey \& Gaston 2002, Petchey et al. 2004 and Ricotta 2005). Due to these shortcomings, new approaches based on the analyses of the trait values of species have been developed to assess the functional richness of communities. The first approach developed along these lines refers to the FR index of Mason et al. (2005) and describes the amount of niche filled by the community (Mason et al. 2005). This index considers only a single functional trait in its computation. However, many studies have demonstrated that considering just one functional trait may induce oversimplified conclusions because the major part of the ecosystemic functions cannot be summarized by a single functional trait (Villéger et al. 2008, Schleuter et al. 2010, Hortal et al. 2015). 
For these reasons, the new approaches developed in recent years for assessing the functional richness of communities are based on simultaneous consideration of several functional traits. Following the definition offered by Mason et al. (2005), the great majority of studies consider functional richness as "the amount of niche space occupied by the species within a community". These approaches take into account the degree of difference among species with reference to functional variables (i.e. functional traits, Petchey \& Gaston 2002, Mason et al. 2003, Mouillot et al. 2005), and aim to estimate their distribution within the community studied. For that purpose, various indices have been developed, based on different mathematical formulas and concepts (e.g. FD index of Petchey \& Gaston 2002, FRic index of Villéger et al. 2008, TOP index of Fontana et al. 2015). Differences between indices arise from the fact that not all authors assess the distribution of species according to their trait values in the same way. Consequently, clustering all these indices under a single general concept (i.e. functional richness) can cause confusion for field ecologists, and misinterpretation of the results obtained.

In this context, the aim of this paper is to clearly identify which index characterizes what (in accordance with their respective mathematical construction), and to highlight how we can use this information to achieve a better - and sometimes more complete - view of the functional richness of a community. On this basis, we propose some guidelines to help users in selecting indices according to the concept of functional richness on which they wish to focus and to the properties of the available data.

More specifically, we focused our discussion on the indices respecting three essential properties in the assessment of the functional richness of communities. Firstly, the index should conserve the continuous values of functional traits (because using functional groups would implicitly assume that species within a group are functionally identical and that species pairs drawn from different groups are equivalent, Ricotta 2005). Secondly, the index should allow the taking into account of several functional traits (to limit the risk of oversimplification and misrepresentation arising from analyses restricted to a single functional trait, Villéger et al. 2008). Thirdly, the index should not take into account the species abundance (to be consistent with the criteria of functional richness as defined by Mason et al. 2005: "a section of niche space is considered to be occupied even if only very little abundance occurs within it").

Consequently, we focused our study on the $F_{P \& G}$ index (Petchey \& Gaston 2002), the FRic index (Villéger et al. 2008), the TOP index (Fontana et al. 2015) and the N-hypervolume index (Blonder et al. 2014), hereafter referred to as 'entities-based approaches'. Furthermore, some recent studies have demonstrated the importance of taking into account the intraspecific trait variability (ITV) in the assessment of the functional richness of communities, because traits vary among genotypes within 
species and according to the environmental conditions (Laughlin \& Laughlin 2013, Messier et al. 2010, Albert et al. 2010, Clark et al. 2011, Auger and Shipley 2013). Thus, we also included in our analyses the FRim index of Schleuter et al. (2010) and the TPD $D_{\text {Rich }}$ index of Carmona et al. (2016a), which are two recent indices taking into account the ITV.

\section{Entities-based approaches}

The indices presented in this section consider as basic unit in their computation the species or the functional entities (i.e. individuals sharing the same combination of trait values).

- The $F D_{P \& G}$ index of Petchey \& Gaston (2002): a dendrogram-based measure

The FD index ( $F D_{P \& G}$; Petchey \& Gaston 2002) has been one of the most widely used indices during the last decades (e.g. Petchey \& Gaston 2006, Flynn et al. 2009, Mendez et al. 2012, Cianciaruso et al. 2013). $F D_{P \& G}$ is based on functional dendrograms, i.e. trees where species are the tips and the length of branches between them reflects the functional dissimilarities between species (Petchey \& Gaston 2002, Maire et al. 2015). This concept is similar to that developed to compute the phylogenetic diversity (Petchey \& Gaston 2002). $F D_{P \& G}$ is the total branch length of the functional dendrogram and consequently, this index measures the overall distance between the different functional units (i.e. all individuals or species sharing the same values of functional traits) of a given community. The greater the distance, the higher the functional richness of this community is considered to be. This index is computed in four steps: (1) obtaining a species * trait matrix (crossing species by their respective trait values), (2) converting the trait matrix into a distance matrix, (3) clustering the distance matrix to produce a dendrogram, and (4) calculating the total branch length of this dendrogram (Petchey \& Gaston 2002). This approach has the advantage of considering the functional difference between species: the more functionally different the species are, the higher the $\mathrm{FD}_{\mathrm{P \& G}}$.

However, the faithfulness of fit between the initial distance matrix (i.e. the reality) and the final dendrogram (i.e. on which the $F D_{P \& G}$ index is based) can vary according to the clustering method (Pielou 1984, Legendre \& Legendre 1998, Podani \& Schmera 2006). More generally, Mouchet et al. (2008) demonstrated that the same combination of distance metric (e.g. Euclidean or Gower metrics) and clustering methods (e.g. UPGMA, UMPMG or Ward clustering) used does not always achieve the best dendrogram. Furthermore, the congruence between the initial distance matrix and the matrix resulting from the classification may decline with a decrease in the number of functional traits and/or of the species richness (Sokal et al. 1992). With the aim of using the most faithful dendrogram to compute the $\mathrm{FD}_{\mathrm{P \& G}}$ of a community, Mouchet et al. (2008) developed an algorithm allowing its identification on the basis of the two-norm quality criterion (which assesses the similarity between the initial dissimilarity matrix and the dissimilarity matrix from different dendrograms, Mérigot et al. 
2010). However, Maire et al. (2015) found that even the 'best' dendrogram (i.e. the most faithful dendrogram) could generate a functional space with a poor fit between the initial and resulting distance matrices. This property could impact our assessment of functional richness and, in consequence, our understanding of ecological functioning and processes (Villéger et al. 2017). Moreover, Schleuter et al. (2010) have also demonstrated that this index could fail to detect some variations in assembly rules.

Given these flaws (i.e. impact of data treatment, impact of the choice of the clustering algorithm, low quality of functional space), measuring functional richness using the $F D_{P \& G}$ index has been considered as hazardous, and may drastically alter the resulting conclusions (Poos et al. 2009). Accordingly, new approaches based on the projection of species in a multidimensional space have been developed.

- The FRic index of Villéger et al. (2008): an index exclusively based on the extreme trait-values The FRic index, named "functional richness" by its developers, represents the amount of functional space filled by the community (Villéger et al. 2008). To avoid confusion between concept and measure, we have called "functional richness" the concept inherent in the definition of Mason et al. (2005, i.e. the amount of functional trait space occupied by the species) and the FRic index, the index developed by Villéger et al. (2008), with the aim of describing this concept.

Mathematically, the FRic index is based on the convex hull concept (i.e. the minimum convex hull which includes all species considered, Cornwell et al. 2006), and corresponds to the volume contained within this hull (Villéger et al. 2008). This volume is computed after projection of each species according to their trait values in a multidimensional space where each dimension represents a functional trait. If categorical traits and/or missing values are present in the initial dataset, a PCoA (Principal Coordinates Analysis) computed from the Gower distance matrix may be used to represent species distribution in a multidimensional functional space (Villéger et al. 2008). Due to the mathematical definition of convex hull volume, only the functional units corresponding to the vertices (i.e. species or individuals with the extreme trait values in the functional space, Cornwell et al. 2006, Villéger et al. 2008) are considered. Thus, in a great majority of cases, FRic considers as filled a potential amount of functional space that is not occupied by any functional units in reality. Consequently, the FRic values for communities that are characterized by species sharing the same extreme trait values are identical, although they may have a very different number of functional units. The Scenario 1 presented in Box 1 illustrates this property. Up to the middle of the simulation, the FRic index values remain identical, despite the intensification of the disturbance. More precisely, FRic index decreases only when the extreme trait-values species begin to be impacted (i.e. from the middle of the simulation process). In this way, FRic will only indicate a disturbance effect on the 
community if the species with extreme trait values are affected (Loiseau et al. 2017). Thus, this index is not well suited for assessing the impact of this type of disturbance (i.e. affecting only the intermediate species) on communities, particularly in the first stages. Moreover, Podani (2009) demonstrated that zero deviation between two convex hulls volumes (e.g. when comparison is made between two communities or a single community at two different periods) does not necessarily mean either that the two convex hulls coincide (i.e. represent a single area of the functional space), or that there is no change in the range and multivariate intersection of traits.

For these reasons, we agree that the FRic index can itself be considered as a functional richness index, but we consider that the computation of this single index cannot be used for summarizing the whole concept of functional richness. FRic is restricted to one facet of the functional richness: the total functional volume occupied by the community (i.e. the volume corresponding to the range of functional traits variability in a given species assemblage, Májeková et al. 2016). Consequently, a better definition for this index seems to be given by Mouillot et al. (2013): "the volume of multidimensional space occupied by all species in a community within functional space", where the functional space is defined as "a multidimensional space where the axes are functional traits [or synthetic combinations of functional traits] along which species are placed according to their functional trait values" (Mouillot et al. 2013).

Furthermore, since every functional unit (here species) represents, by definition, a unique combination of traits, all species should be taken into consideration when calculating the functional richness of a community (Fontana et al. 2014, Fontana et al. 2015), and not only the vertices species. On the basis of this latter point, Fontana et al. (2015) considered that the FRic index was not wellsuited for the assessment of the functional richness of a community, and they have proposed a new index, the TOP index, to remedy this.

- The TOP index of Fontana et al. (2015): taking into account all functional units

In contrast to FRic, the TOP index ("Trait Onion Peeling", Fontana et al. 2015) aims "to comprehensively describe changes in the internal structure of multidimensional trait space". Since it considers all functional units (individuals/species) in the community, the TOP index is intended to minimize the overestimation of the filled functional space. These authors considered that TOP corresponds to the amount of functional space filled by the community.

As for FRic, the computation of TOP is based on the construction of convex hulls, which represent the smallest convex set enclosing all the points of a dataset, regardless of the shape of the distribution pattern (Cornwell et al. 2006). While FRic is computed on the basis of a single convex hull, the TOP index is based on the sum of successive convex hulls. Firstly, all individuals (or species) within a community are projected in the functional space according to their functional trait values. Secondly, 
the first minimum convex hull containing the outermost points (e.g. individuals) of functional space is built and its area is measured. Thirdly, these points are deleted from the trait distribution and a second convex hull is calculated with the new outermost points. This process continues, as "if peeling off the layers of an onion", until the number of remaining points is insufficient for computing a convex hull (Fontana et al. 2015). The sum of all areas (convex hulls) obtained in this way represents the TOP index (see Fontana et al. 2015). The TOP index describes both the overall range and composition of functional space in terms of phenotypes and trait combinations (including both extreme and intermediate species, Fontana et al. 2015). However, TOP index corresponding to the sum of all successive convex hulls, is more sensitive to disturbances that impact the extreme trait values species than the species with mean (or intermediate) trait values (because the volume represented by the convex hull of the extreme trait values species is higher). The comparison of the trend curves of TOP index across the three scenarios in Box 1 illustrates this property. In Scenario 1 (which is the only one where species which are affected in the first stages of a disturbance are not extreme trait values species), the TOP index tends to decreases slower than in the two other scenarios. This is because the volume that is subtracted at each step time comes from intermediate trait values species, and not from extreme trait values species (and by consequence, this volume is smaller). Thus, the TOP index performs weakly to detect the first stages of a disturbance that would affect functionally intermediate trait values species. On the other hand, the results of scenario 1 highlights that it is still preferable to use the TOP index rather than the FRic index to assess the effect of this type of disturbance (i.e. which mainly affects intermediate trait values species) on the communities because the TOP index detects the disturbance more quickly (Figure 1 in Box 1). However, the TOP index requires a long computation time that could make it unusable when the dimensionality of the data is too high (see Ross et al. 2017 for an example of this situation).

- The N-dimensional hypervolume (N-Hyp.) index of Blonder et al. (2014): taking into account disjunctions and holes.

The two previous indices (the FRic and TOP indices) are based on the estimation of convex hull volumes. However, the convex hulls method suffers from two major limitations. Firstly, approaches based on this concept are highly sensitive to the outlier points (vertices species), particularly the FRic index, and secondly, they cannot model (i.e. take into account) disjunctions or holes in functional space filled by the community (Blonder et al. 2014).

To remedy these flaws, Blonder et al. (2014) developed a new method based on the estimation of hypervolumes from observations in $\mathrm{n}$-dimensions. In contrast to the other methods, their framework accounts for disjunctions or holes, and it is not sensitive to outlier points. We can resume this 
framework in the following way (see also Fig 1). They firstly compute a KDE (Kernel Density Estimator) with a bandwidth $\vec{h}$ (determining the width of the kernel functions) around each initial observation (i.e. around each species present in the functional space after projection according to functional trait values). Then, they estimate the distribution of probabilistic functions obtained and they slice this distribution until at least the chosen fraction 1- $\tau$ of the probability density is enclosed by the distribution (where $\tau$ corresponds to a quantile threshold chosen by the user that is within the range 0 to 1). The $\mathrm{N}$-hypervolume index is computed from this truncated distribution pattern (see Blonder et al. 2014 for more details on the mathematical properties) and the extension of this method to multiple dimensions). Although Blonder et al. (2014) asked users to report the chosen bandwidth $\vec{h}$ and the quantile $\tau$ chosen with the aim of ensuring that hypervolume analyses are replicable, they recognize that these two subjective choices could impact the result. In a general way, choosing a smaller bandwidth and/ or a larger threshold will lead to a smaller hypervolume (because each observation appears more disjointed from the others), while choosing a larger bandwidth (or smaller threshold) for the same dataset will lead to a larger volume (because there are more observations appearing to be connected, Blonder et al. 2014).

Moreover, Qiao et al. (2016) have demonstrated that the KDE (i.e. the method used to estimate the $\mathrm{n}$-dimensional hypervolume) only recognizes clusters or holes when occurrence data are numerous (i.e. higher than 100 for clusters and higher than 1000 for holes). Because many studies assessed communities with a smaller number of species, this property could be problematic in community ecology (e.g. Villéger et al. 2010, Luo et al. 2016, Cianciaruso et al. 2013). These authors have also shown that KDE may overestimate the hypervolume in low dimensions (i.e. lower than four dimensions where each dimension corresponds to a functional trait or to a combination of functional traits) and underestimate it in high dimensions (i.e. higher than six dimensions). Thus, this index may both under- and overestimate niche volumes, depending on the dimensionality of the data and the number of occurrence records considered (Qiao et al. 2016).

This phenomenon is directly illustrated in our study in Scenario 1, presented in Box 1. In this scenario, the $\mathrm{N}$-hypervolume index increases while the disturbance is increasing, which is counter-intuitive for an index supposed to assess functional richness. We can explain this unexpected behaviour by the mathematical properties of the Silverman bandwidth estimator (determining the width of the kernel functions and defined as $1.06 * s d(X) * m^{(-1 / 5)}$ where $m$ is the number of observations and $X$ is the data vector). In Scenario 1, $m$ decreases whereas the standard deviation (i.e. $s d(X)$ ) increases, which induces an increase in the bandwidth parameter and, in consequence, an overestimation of the hypervolume computed. This effect is counteracted in Scenario 2 and Scenario 3 due to the decrease 
of the standard deviation of the trait values for only one functional trait (Scenario 2) or all functional traits (Scenario 3).

With the aim of considering disjunctions and holes inside the functional space, the approach developed by Blonder et al. (2014) represents a real advance in the estimation of the functional space filled by the community. However, some important issues remain, such as the impact of the choice of the two parameters required for the computation of the n-hypervolumes (notably the bandwidth vector) or its capacity to detect holes according to the dimensions of the functional space and the size of the dataset.

\section{Taking into account the intraspecific trait variability: the emergence of new approaches}

In this work, we consider the taking into account of intraspecific trait variability (ITV) as the fact that a functional trait is considered as a random variable drawn from a probability distribution (Albert et al. 2011). Following this definition, none of the four indices considered above really takes into account the intraspecific trait variability. The use of functional richness indices taking into account ITV in the scientific literature is still very scarce, in particular because of the poor availability of the corresponding information. However, this situation is progressively changing for numerous taxa, and this trend is likely to continue and to further develop in the years to come (Carmona et al. 2016a). In fact, an increasing number of studies have demonstrated that the ITV should not be neglected in the assessment of different aspects of functional diversity (Violle et al. 2007, Albert et al. 2012, Laughlin et al. 2015, Gonzales-Suarez 2015). The ITV can explain a significant part of the functional trait variability within communities and can have a strong impact on population stability, species coexistence and ecosystem processes (Bolnick et al. 2011, Kraft et al. 2014, Cochrane et al. 2015, Carmona et al. 2016a). With the aim of taking into account this variation in the assessment of functional richness, two main methods have been developed to date.

- The FRim index of Schleuter et al. (2010)

The first index established to take into account the ITV and the probabilistic nature of functional traits in the assessment of functional richness of communities is the FRim index of Schleuter et al. 2010. In the description of their index, the authors state that FRim aims to consider the ITV and the gaps in functional space. The mathematical construction of this index can be summarized as follows. The general idea is to assign to each species $i$ a membership function that describes its distribution in functional space. This membership function is in the range between 0 et 1 . The closer this value is to one, the higher the probability that the point of functional space considered belongs to the species $i$. Then, the total volume of trait space occupied by a community is obtained by computing the integral 
of the maximum of all species membership functions (Schleuter et al. 2010). This volume corresponds to the FRim index value (see Schleuter et al. 2010 for more details regarding the computation of the FRim index). In their study, Schleuter et al. 2010 suggest considering a function, called $f s$, that uses information on both mean trait values and trait variance-covariance matrices to establish the membership functions of species (these matrices are computed from the trait values of all individuals belonging to each species). The FRim index is then obtained as the integral of the maximum of all $f s$ functions (integrated over the studied trait space, see Schleuter et al. 2010 for further details). Thus, this approach does not take into account the space occupied by a species within the functional space on the basis of a single point (but by taking into account the whole surface area covered by its $f s$ function).

However, computing the functional space occupied by each species on the basis of a membership function integrating the trait mean value and the trait variance value could lead to an estimation of functional richness strongly linked to the size of the dataset considered. Let us consider three assemblages of individuals ( $11, A 2$ and $A 3$ ), each composed of a single species and by 3,5 and 7 individuals respectively. The individual values for a functional trait are respectively $A 1=[3.5,4,4.5]$, $A 2=[3.5,3.75,4,4.25,4.5]$ and $A 3=[3.5,3.75,3.9,4,4.1,4.25,4.5]$. For these three assemblages, both the mean trait values (i.e. 4) and the range trait values ( 3.5 to 4.5 ) are the same, but, due to the difference in vector length (abundance), their trait variance values differ (VarA1 > VarA2 > VarA3). In consequence, the integrate values of their respective membership functions differ as well as, in fine, the FRim index value. The FRim (corresponding to the integral of the $f s(x)$ function for each assemblage) would be greater for A1 than for A2, and the latter would be greater than A3 (see Figure 2 for illustration). This property could seem to be counter-intuitive, because we could instinctively consider that functional richness of $A 3$ is the highest and the functional richness of $A 1$ is the lowest (because the number of functional units is lower in $A 1$ than in $A 3$ and that the range of trait values covered by these two communities is the same). It is then impossible to know whether the FRim index value for $A 1$ is a real overestimation of the functional space occupied by the community, or an accurate inference of missing individual trait values due to insufficient sampling. It is also important to note that for the sake of simplicity, this property is illustrated here with a very simple example (i.e. one functional trait and one species), but it remains valid for assemblages that are more complex (i.e. with more species and more functional traits).

Furthermore, as pointed out by its developers, this index requires a long computation time (Schleuter et al. 2010). This long computation time is exacerbated when the number of observations and/or the number of functional traits is high. Finally, due to its concept of computation, FRim necessarily requires taxonomic classification of the different individuals at the species level. The 
latter point could be problematic in studies where the organisms studied are difficult to identify up to species level (e.g. phytoplankton, Fontana et al. 2014).

\section{- The TPD Rich: TPD framework of Carmona et al. (2016a)}

The second framework taking into account this intra-variability of species was developed by Carmona et al. (2016a), and called the TPD Rich (trait probability density for richness component) framework. This framework is based on the assumption that not all combinations of functional traits confer equal fitness to the functional units considered. For example, according to their values of functional traits, not all individuals of a same species have the same probability of survival and reproduction. This implies that the trait values within a given functional unit (here a species) are not equally represented across the whole range of possible values. In consequence, these authors have developed a new approach taking into account the probabilistic nature of functional traits. For this, they expressed functional niches as probability density functions, where the values of these functions for each functional unit are directly proportional to their relative abundance.

More specifically, they assigned to each individual of each species a $\operatorname{TPD}_{i}$ function (integrate to 1 ) that represents the probability values for a given functional trait. All these TPD (i.e. TPD at the individual level) are then summed to obtain the trait distribution probability for each species (TPDs, see Carmona et al. (2016a) for more details on the computation of the TPD framework). This framework has been developed in order to quantify various components of the functional diversity. One part of this general framework is specifically focused on the assessment of the functional richness. The functional richness is here considered as the amount of functional volume occupied by all $\mathrm{TPD}_{\mathrm{S}}$ of the community, which can be estimated as the sum of the hypervolumes of the cells where $T P D_{S}$ is greater than zero. In this way, the use of TPD functions enables estimation of the functional richness incorporating the ITV and conservation of the original probabilistic context of functional trait values (Mason et al. 2005, Carmona et al. 2016a). However, using this framework requires having a significant number of observations for all functional units in a sampling (in order to be able to compute the realistic probabilistic trait values). The greater the numbers of observations, the closer the probabilistic functions of trait values are to reality. However, given the constraints and limitations of field studies in numerous domains, it can be very difficult - and sometimes impossible to sample the required amount of observations. Moreover, the TPD framework also used the KDE method to estimate the intraspecific trait intra-variability, and in consequence, the same problems as those observed for the n-hypervolume index could occur (i.e. under or over-estimation of the occupied volume). 
Furthermore, in a response to the paper of Carmona et al. (2016a), Blonder (2016) raised some concerns regarding the TPD framework. Firstly, this author highlighted that the use of the probability function with non-zero density everywhere and no defined thresholds could lead to hypervolumes with infinite boundaries (and in consequence, not available for the analysis). He also demonstrated that the choice of this threshold could lead to different values of functional richness and in consequence, to differences in the interpretation of the results obtained. Secondly, he stated that the overlap between two distributions is strongly dependent on the width of the trait distributions (i.e. of the $T P D_{s}$ ). The overlap between a wide distribution and a distribution where trait values are concentrated in a particular part of its range will be always low (Blonder 2016). To overcome this drawback, Blonder (2016) suggested integrating all trait distributions to one, but this suggestion was rejected by Carmona at al. (2016b), considering that is preferable to let the distribution of density be wholly defined by the real data (to avoid missing certain ecological phenomena).

\section{DISCUSSION}

Mason et al. (2005) originally defined functional richness, which is one of three major components of functional diversity, as "the amount of functional trait space occupied by the species". Based on this definition, a range of indices has been developed with the aim of assessing this component. However, although the authors argued that their indices describe the functional richness, we have demonstrated here that each of them describes only a part of it. A detailed survey clearly shows that all of these indices have different advantages and drawbacks, and, more importantly, describe different aspects of functional richness. In the entities-based approaches (i.e. approaches that do not consider the intraspecific trait variability), we can distinguish two principal frameworks: one based on dendrograms with the $F D_{P \& G}$, and another based on multidimensional methods with the FRic, TOP and $\mathrm{N}$-hypervolume indices. The main issue of the first framework represented by the $F D_{P \& G}$ index is that the clustering algorithm used to build the dendrogram arbitrarily affects its final representation and, in consequence, the values of the $\mathrm{FD}_{P \& G}$ index (Podani \& Schmera 2006). Accordingly, indices using multidimensional functional spaces (i.e. functional space where species are plotted along trait axes, or synthetic axes capturing combined traits, Villéger et al. 2008), have been proposed more recently. Although use of ordination methods can also distorted the functional space, this distortion remains smaller than those induced by the clustering methods (Maire et al. 2015). These indices are the FRic, TOP and N-hypervolume indices. However, even if these three indices belong to the same mathematical approach (i.e. conservation of multidimensionality of functional space), some crucial differences are observed. More specifically, we showed in this study that these three indices do not respond in the same way to the different types of disturbances affecting the communities. For example, we demonstrated that for a disturbance particularly affecting the species located in the 
centre of functional space, the $\mathrm{N}$-hypervolume index has a counter-intuitive behaviour (i.e. it increases with the intensification of the disturbance) due to a mathematical artefact. In the same vein, the FRic index (and to a lesser extent, the TOP index) begin to detect the impact of this type of disturbance after an long time lapse because it takes into account only the extreme trait values species in its computation. Consequently, according to the nature of the disturbance forcing the community, the user should not choose the same index for assessing the changes of functional richness of the community. Furthermore, due to the growing consensus within the scientific community that the intraspecific trait variability (ITV) has a strong impact on population stability, species coexistence and ecosystem processes (Violle et al. 2007, Carmona et al. 2016a), new methodological approaches have emerged to take into account this variability, based on the probabilistic nature of functional traits. These approaches refer to the FRim index (Schleuter et al. 2010) and the TPD framework (Carmona et al. 2016a). However, both approaches developed require a large number of observations for each species (in order to have a correct estimation of the species mean trait values and of their respective distribution), that is not always feasible in the face of the technical constraints encountered in reality. In numerous cases, the fieldwork constraints (generally linked to time and/or cost constraints) limit our capacity to produce an accurate census for many organisms (Hortal et al. 2015, Ross et al. 2017). In this case, a feasible alternative way for incorporating ITV could be through a return to entities-based indices. It might be possible to incorporate some intraspecific trait information within these indices. The functional matrix required for these approaches can be considered both at the individual and species level (i.e. in considering an individual * trait matrix instead of species * trait matrix, Fontana et al. 2015). Furthermore, Ross et al. (2017) have recently developed a new method to incorporate the ITV into the existing approaches. This approach is essentially based on a simulation procedure and offers a basis for dealing with case studies where individual data are not available for all functional traits and/or all individuals of each species (see Ross et al. 2017 for details on the simulation process).

Thus, all the indices and methods reviewed in this study may be used for the purpose of assessing functional richness, but most of them do not assess the same part of this component, which is far more complex than expected. With the aim of clearly distinguishing between the terminology used and the concept itself, we have summarized in Table 1 what each index really measures, as well as their respective advantages and drawbacks. In addition, we should keep in mind that some authors (Schleuter et al. 2010, Blonder et al. 2016) have pointed out some issues resulting from the consideration of categorical traits (nominal and/or ordinal) in the computation of the majority of indices used in the assessment of functional richness. These authors highlighted the fact that categorical traits should be handled with care, and that the metric distance used in the computation 
of the indices should satisfy the triangle inequality (such as the Gower dissimilarity, Blonder et al. 2016). Despite this precaution, Schleuter et al. (2010) suggested avoiding their use because the transformation of categorical variables into numerous variables (through a principal component analysis, or Hill and Smith's method) could affect the structure of the original dataset. However, this recommendation is difficult to apply in the real world because a large number of studies use categorical variables for large numbers of taxa (e.g. Flynn et al. 2009, Carboni et al. 2013, Forrest et al. 2015, Loiseau et al. 2016). Excluding this family of traits in the assessment of functional richness would lead to a serious loss of functional information and, consequently, to a serious underestimation of the functional diversity. Thus, rather than excluding this category of variables, we would recommend always checking the validity of the transformation realized on the initial dataset before attempting any interpretation of the results obtained (e.g. with the mSD index developed by Maire et al. 2015).

Our study also highlights that the multicomponent basis of the functional richness concept clearly implies that using one index rather than another is not without significance. Indeed, this choice implies focusing on some aspects of functional richness, but ignoring others. Thus, the main question which the users must address is "What facet of functional richness do we really want to assess?": is it the functional distance between species, the functional range covered by the community, or the volume of functional space filled by the community ? It is to a large extent the answer to this question that determines the index to be selected. If the goal of the study is to estimate the functional distance between species, only the $F D_{P \& G}$ is suitable. Similarly, if the user is interested in measuring the total functional range covered by the community, only the FRic index is suitable. On the other hand, if the user needs to estimate how much of the functional space is filled, several choices are possible. The FRim or the TDP $_{\text {Rich }}$ indices may be chosen if the intraspecific variability in trait values is available, whereas the N-hypervolume or the TOP indices could be chosen if not. Thus, with the aim of clearly identifying the differences of concept between the different indices, we provide some guidelines in Figure 3 that, in complement to the information offered in Table 1, can guide the ecologists in the choice of indices according to the goal of each study. Such knowledge is essential both for choosing indices that properly match the question addressed, and for properly interpreting the results obtained.

To conclude, we have shown that functional richness is a far more complex component than usually suggested. This component encompasses different concepts that can be assessed on the basis of an increasing range of indices. Defining which indices should be used and which indices should be avoided is a complex issue. Through an in-depth analysis of the existing approaches and of their respective concepts, we hope that our work could help ecologists in this arduous, but necessary, step 
in any scientific study. Finally, we join Schmera et al. (2015) in arguing that researchers should clearly separate concept and measure in order to avoid any confusion in the interpretation of results, and in consequence, in the resulting conclusions. This latter point is of major importance at a time when the functional richness is taken into account as a basis for decision making in the management and conservation plans (Vitule et al. 2017).

\section{ACKNOWLEDGMENTS}

This work was supported by the University of French Polynesia (ED 469) and funded by the Research Ministry of French Polynesia (Délégation à la Recherche de Polynesie Française). We are grateful to M. Paul for improvements to the English text.

\section{REFERENCES}

Albert, C. H., de Bello, F., Boulangeat, I., Pellet, G., Lavorel, S., \& Thuiller, W. (2012). On the importance of intraspecific variability for the quantification of functional diversity. Oikos, 121(1), 116-126.

Albert, C. H., Grassein, F., Schurr, F. M., Vieilledent, G., \& Violle, C. (2011). When and how should intraspecific variability be considered in trait-based plant ecology?. Perspectives in Plant Ecology, Evolution and Systematics, 13(3), 217-225.

Auger, S., \& Shipley, B. (2013). Inter-specific and intra-specific trait variation along short environmental gradients in an old-growth temperate forest. Journal of Vegetation Science, 24(3), 419-428.

Blondel, J. (2003). Guilds or functional groups: does it matter?. Oikos, 100(2), 223-231.

Blonder, B. (2016). Pushing past boundaries for trait hypervolumes: A Response to Carmona et al. Trends in ecology \& evolution, 31(9), 665-667.

Blonder, B., Lamanna, C., Violle, C., \& Enquist, B. J. (2014). The n-dimensional hypervolume. Global Ecology and Biogeography, 23(5), 595-609.

Bolnick, D. I., Amarasekare, P., Araújo, M. S., Bürger, R., Levine, J. M., Novak, M., ... \& Vasseur, D. A. (2011). Why intraspecific trait variation matters in community ecology. Trends in ecology \& evolution, 26(4), 183-192.

Carboni, M., Acosta, A. T., \& Ricotta, C. (2013). Are differences in functional diversity among plant communities on Mediterranean coastal dunes driven by their phylogenetic history?. Journal of Vegetation Science, 24(5), 932-941.

Carmona, C. P., de Bello, F., Mason, N. W., \& Lepš, J. (2016a). Traits without borders: integrating functional diversity across scales. Trends Ecol Evol, 31, 382-94. 
Carmona, C. P., de Bello, F., Mason, N. W., \& Lepš, J. (2016b). The density awakens: a reply to Blonder. Trends in ecology \& evolution, 31(9), 667-669.

Chapin III, F. S., Reynolds, H. L., D'Antonio, C. M., \& Eckhart, V. M. (1996). The functional role of species in terrestrial ecosystems. Global change and terrestrial ecosystems, 403, 403-406.

Cianciaruso, M. V., Batalha, M. A., \& Petchey, O. L. (2013). High loss of plant phylogenetic and functional diversity due to simulated extinctions of pollinators and seed dispersers in a tropical savanna. Natureza \& Conservação, 11, 36-42.

Clark, J. S., Bell, D. M., Hersh, M. H., Kwit, M. C., Moran, E., Salk, C., ... \& Zhu, K. (2011). Individualscale variation, species-scale differences: inference needed to understand diversity. Ecology Letters, 14(12), 1273-1287.

Cochrane, A., Yates, C. J., Hoyle, G. L., \& Nicotra, A. B. (2015). Will among-population variation in seed traits improve the chance of species persistence under climate change?. Global Ecology and Biogeography, 24(1), 12-24.

Cornwell, W. K., Schwilk, D. W., \& Ackerly, D. D. (2006). A trait-based test for habitat filtering: convex hull volume. Ecology, 87, 1465-1471.

Diaz, S., and Cabido, M. (2001). Vive la difference: plant functional diversity matters to ecosystem processes. Trends Ecol. Evol. 16, 646-655.

Flynn, D. F., Gogol-Prokurat, M., Nogeire, T., Molinari, N., Richers, B. T., Lin, B. B., ... \& DeClerck, F. (2009). Loss of functional diversity under land use intensification across multiple taxa. Ecology letters, 12(1), 22-33.

Fontana, S., Jokela, J., \& Pomati, F. (2014). Opportunities and challenges in deriving phytoplankton diversity measures from individual trait-based data obtained by scanning flowcytometry. Frontiers in microbiology, 5.

Fontana, S., Petchey, O. L., \& Pomati, F. (2015). Individual-level trait diversity concepts and indices to comprehensively describe community change in multidimensional trait space. Functional Ecology. doi: 10.1111/1365-2435.12551

Forrest, J. R., Thorp, R. W., Kremen, C., \& Williams, N. M. (2015). Contrasting patterns in species and functional-trait diversity of bees in an agricultural landscape. Journal of Applied Ecology, 52(3), 706-715.

González-Suárez, M., Bacher, S., \& Jeschke, J. M. (2015). Intraspecific trait variation is correlated with establishment success of alien mammals. The American Naturalist, 185(6), 737-746.

Hector, A., Schmid, B., Beierkuhnlein, C., Caldeira, M. C., Diemer, M., Dimitrakopoulos, P. G., ... \& Harris, R. (1999). Plant diversity and productivity experiments in European grasslands. science, 286(5442), 1123-1127. 
Holmes, R. T., Bonney, R. E., \& Pacala, S. W. (1979). Guild structure of the Hubbard Brook bird community: a multivariate approach. Ecology, 60(3), 512-520.

Hortal, J., de Bello, F., Diniz-Filho, J. A. F., Lewinsohn, T. M., Lobo, J. M., \& Ladle, R. J. (2015). Seven shortfalls that beset large-scale knowledge of biodiversity. Annual Review of Ecology, Evolution, and Systematics, 46, 523-549.

Kraft, N. J., Crutsinger, G. M., Forrestel, E. J., \& Emery, N. C. (2014). Functional trait differences and the outcome of community assembly: an experimental test with vernal pool annual plants. Oikos, 123(11), 1391-1399.

Krismer, J., Tamminen, M., Fontana, S., Zenobi, R., \& Narwani, A. (2016). Single-cell mass spectrometry reveals the importance of genetic diversity and plasticity for phenotypic variation in nitrogen-limited Chlamydomonas. The ISME journal.

Lamanna, C., Blonder, B., Violle, C., Kraft, N. J., Sandel, B., Šímová, I., ... \& Buzzard, V. (2014). Functional trait space and the latitudinal diversity gradient. Proceedings of the National Academy of Sciences, 111(38), 13745-13750.

Laughlin, D. C., \& Laughlin, D. E. (2013). Advances in modeling trait-based plant community assembly. Trends in plant science, 18(10), 584-593.

Laughlin, D. C., \& Messier, J. (2015). Fitness of multidimensional phenotypes in dynamic adaptive landscapes. Trends in Ecology \& Evolution, 30(8), 487-496.

Lavorel, S., \& Garnier, É. (2002). Predicting changes in community composition and ecosystem functioning from plant traits: revisiting the Holy Grail. Functional ecology, 16(5), 545-556.

Legendre, P., \& Legendre, L. (1998). Numerical ecology: second English edition. Developments in environmental modelling, 20.

Loiseau, N., Legras, G., Gaertner, J. C., Verley, P., Chabanet, P., \& Mérigot, B. (2017). Performance of partitioning functional beta-diversity indices: Influence of functional representation and partitioning methods. Global Ecology and Biogeography, 26(6), 753-762.

Loiseau, N., Legras, G., Kulbicki, M., Mérigot, B., Harmelin-Vivien, M., Mazouni, N., ... \& Gaertner, J. C. (2016). Multi-component $\beta$-diversity approach reveals conservation dilemma between species and functions of coral reef fishes. Journal of Biogeography.

Luo, Y., Liu, J., Tan, S., Cadotte, M. W., Xu, K., Gao, L., \& Li, D. (2016). Trait variation and functional diversity maintenance of understory herbaceous species coexisting along an elevational gradient in Yulong Mountain, Southwest China. Plant Diversity, 38(6), 303-311.

Maire, E., Grenouillet, G., Brosse, S., and Villéger, S. (2015). How many dimensions are needed to accurately assess functional diversity? a pragmatic approach for assessing the quality of functional spaces. Global Ecology and Biogeography. 
Májeková, M., Paal, T., Plowman, N. S., Bryndová, M., Kasari, L., Norberg, A., ... \& Le BagoussePinguet, Y. (2016). Evaluating Functional Diversity: Missing Trait Data and the Importance of Species Abundance Structure and Data Transformation. PloS one, 11(2), e0149270.

Mason, N. W., MacGillivray, K., Steel, J. B., \& Wilson, J. B. (2003). An index of functional diversity. Journal of Vegetation Science, 14(4), 571-578.

Mason, N.W., Mouillot, D., Lee, W.G., and Wilson, J.B. (2005). Functional richness, functional evenness and functional divergence: the primary components of functional diversity. Oikos 111, 112-118.

Mendez, V., Gill, J. A., Burton, N. H., Austin, G. E., Petchey, O. L., \& Davies, R. G. (2012). Functional diversity across space and time: trends in wader communities on British estuaries. Diversity and Distributions, 18(4), 356-365.

Mérigot, B., Durbec, J. P., \& Gaertner, J. C. (2010). On goodness-of-fit measure for dendrogram-based analyses. Ecology, 91(6), 1850-1859.

Messier, J., McGill, B. J., \& Lechowicz, M. J. (2010). How do traits vary across ecological scales? A case for trait-based ecology. Ecology letters, 13(7), 838-848.

Mouchet, M., Guilhaumon, F., Villéger, S., Mason, N. W., Tomasini, J. A., \& Mouillot, D. (2008). Towards a consensus for calculating dendrogram-based functional diversity indices. Oikos, 117, 794-800.

Mouillot, D., Graham, N. A., Villéger, S., Mason, N. W., \& Bellwood, D. R. (2013). A functional approach reveals community responses to disturbances. Trends in Ecology \& Evolution, 28(3), 167-177.

Mouillot, D., Mason, W. N., Dumay, O., \& Wilson, J. B. (2005). Functional regularity: a neglected aspect of functional diversity. Oecologia, 142(3), 353-359.

Petchey, O. L., \& Gaston, K. J. (2002). Functional diversity (FD), species richness and community composition. Ecology Letters, 5, 402-411.

Petchey, O.L., and Gaston, K.J. (2006). Functional diversity: back to basics and looking forward. Ecology Letters 9, 741-758.

Petchey, O.L., Hector, A., and Gaston, K.J. (2004). How do different measures of functional diversity perform? Ecology 85, 847-857.

Pielou, E. C. (1984). The interpretation of ecological data: a primer on classification and ordination. John Wiley \& Sons.

Podani, J. (2009). Convex hulls, habitat filtering, and functional diversity: mathematical elegance versus ecological interpretability. Community Ecology, 10(2), 244-250.

Podani, J., and Schmera, D. (2006). On dendrogram-based measures of functional diversity. Oikos, $115,179-185$. 
Poos, M. S., Walker, S. C., \& Jackson, D. A. (2009). Functional-diversity indices can be driven by methodological choices and species richness. Ecology, 90(2), 341-347.

Qiao, H., Escobar, L. E., Saupe, E. E., Ji, L., \& Soberón, J. (2016). A cautionary note on the use of hypervolume kernel density estimators in ecological niche modelling. Global Ecology and Biogeography.

Reich, P. B., Tilman, D., Craine, J., Ellsworth, D., Tjoelker, M. G., Knops, J., ... \& Bengtson, W. (2001). Do species and functional groups differ in acquisition and use of $\mathrm{C}, \mathrm{N}$ and water under varying atmospheric $\mathrm{CO} 2$ and $\mathrm{N}$ availability regimes? A field test with 16 grassland species. New Phytologist, 150(2), 435-448.

Ricotta, C. (2005). A note on functional diversity measures. Basic and Applied Ecology 6, 479-486.

Ross, S. R. J., Hassall, C., Hoppitt, W. J., Edwards, F. A., Edwards, D. P., \& Hamer, K. C. (2017). Incorporating intraspecific trait variation into functional diversity: Impacts of selective logging on birds in Borneo. Methods in Ecology and Evolution.

Schleuter, D., Daufresne, M., Massol, F., and Argillier, C. (2010). A user's guide to functional diversity indices. Ecological Monographs 80, 469-484.

Schleuter, D., Daufresne, M., Veslot, J., Mason, N. W., Lanoiselée, C., Brosse, S., ... \& Argillier, C. (2012). Geographic isolation and climate govern the functional diversity of native fish communities in European drainage basins. Global Ecology and Biogeography, 21(11), 10831095.

Schmera, D., Podani, J., Heino, J., Erős, T., \& Poff, N. L. (2015). A proposed unified terminology of species traits in stream ecology. Freshwater Science, 34(3), 823-830.

Sokal, R. R., Kim, J., \& Rohlt, F. J. (1992). Character and OTU stability in five taxonomic groups. Journal of classification, 9(1), 117-140.

Tilman, D., Knops, J., Wedin, D., Reich, P., Ritchie, M., \& Siemann, E. (1997). The influence of functional diversity and composition on ecosystem processes. Science, 277(5330), 13001302.

Villéger, S., Maire, E., \& Leprieur, F. (2017). On the risks of using dendrograms to measure functional diversity and multidimensional spaces to measure phylogenetic diversity: a comment on Sobral et al.(2016). Ecology Letters, 20(4), 554-557.

Villéger, S., Mason, N.W.H., and Mouillot, D. (2008). New multidimensional functional diversity indices for a multifaceted framework in functional ecology. Ecology 89, 2290-230

Villéger, S., Miranda, J. R., Hernández, D. F., \& Mouillot, D. (2010). Contrasting changes in taxonomic vs. functional diversity of tropical fish communities after habitat degradation. Ecological Applications, 20(6), 1512-1522. 
Violle, C., Navas, M. L., Vile, D., Kazakou, E., Fortunel, C., Hummel, I., \& Garnier, E. (2007). Let the concept of trait be functional!. Oikos, 116(5), 882-892.

Vitule, J. R., Agostinho, A. A., Azevedo-Santos, V. M., Daga, V. S., Darwall, W. R., Fitzgerald, D. B., ... \& Orsi, M. L. (2017). We need better understanding about functional diversity and vulnerability of tropical freshwater fishes. Biodiversity and Conservation, 26(3), 757-7 


\section{TABLES}

Table 1: Definitions, main advantages and drawbacks of functional richness indices considered in this study. ITV: Intraspecific trait variability; TPD: Trait probability density. FU: Functional unit (i.e. all individuals or species sharing the same values of functional traits). For the References column, $\left({ }^{*}\right)$ means that the corresponding study uses the TOP index of Fontana et al. (2015) not to assess the phenotypic variation of communities (and not to assess the functional richness), and $\left({ }^{* *}\right.$ ) means that this study uses the index of Schleuter et al. (2010) in a one-dimensional context. No other studies are found for the two approaches. Nevertheless, probably due to the recent character of the approach developed by Carmona et al. 2016, no case study using this method has been found.

\begin{tabular}{|c|c|c|c|c|c|}
\hline Index & Formula & Measures of... & Advantages & Drawbacks & References \\
\hline $\begin{array}{l}\text { FD } D_{P \& G} \\
\text { (Petchey and } \\
\text { Gaston, 2002) }\end{array}$ & $\begin{array}{l}\text { Total branch } \\
\text { length of the } \\
\text { functional } \\
\text { dendrogram }\end{array}$ & $\begin{array}{l}\text { Overall } \\
\text { distance } \\
\text { between } \\
\text { different } \\
\text { functional units }\end{array}$ & $\begin{array}{ll}-\quad \text { Taking into } \\
\text { account all } \\
\text { species } \\
\text { No constraints on } \\
\text { the number of } \\
\text { species and } \\
\text { number of traits }\end{array}$ & $\begin{array}{ll}- & \text { Clustering } \\
\text { methods can } \\
\text { impact the result } \\
\text { - } \quad \text { Dendrogram- } \\
\text { based functional } \\
\text { space could be of } \\
\text { poor quality }\end{array}$ & $\begin{array}{l}\text { - } \quad \text { Flynn et al. } \\
2009\end{array}$ \\
\hline $\begin{array}{l}\text { FRic } \\
\text { (Villéger et al. } \\
2008 \text { ) }\end{array}$ & $\begin{array}{l}\text { Smallest } \\
\text { convex hull } \\
\text { containing all } \\
\text { species }\end{array}$ & $\begin{array}{l}\text { Volume of } \\
\text { functional } \\
\text { space occupied } \\
\text { by all species }\end{array}$ & $\begin{array}{l}\text { - Keeps the notion } \\
\text { of } \\
\text { multidimensionali } \\
\text { ty of functional } \\
\text { space } \\
\text { - Not sensitive to } \\
\text { the species } \\
\text { richness }\end{array}$ & 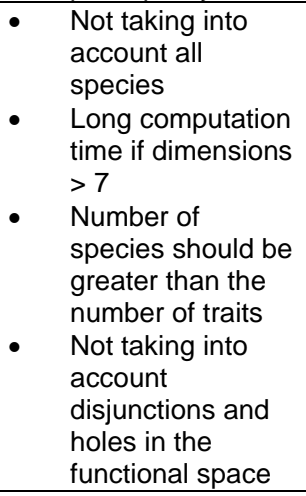 & $\begin{array}{l}\text { - } \quad \text { Luo et al. } \\
2016\end{array}$ \\
\hline $\begin{array}{l}\text { TOP } \\
\text { (Fontana et al. } \\
2015 \text { ) }\end{array}$ & $\begin{array}{l}\text { Sum of areas of } \\
\text { successive } \\
\text { convex hulls }\end{array}$ & $\begin{array}{l}\text { Overall range } \\
\text { and } \\
\text { composition of } \\
\text { functional } \\
\text { space }\end{array}$ & $\begin{array}{l}\text { - Taking into } \\
\text { account all } \\
\text { species } \\
\text { Minimizes the } \\
\text { overestimation of } \\
\text { the filled } \\
\text { functional space }\end{array}$ & $\begin{array}{l}\text { - Long computation } \\
\text { time } \\
\text { Number of } \\
\text { species should be } \\
\text { greater than the } \\
\text { number of traits } \\
\text { Not taking into } \\
\text { account } \\
\text { disjunctions and } \\
\text { holes in the } \\
\text { functional space }\end{array}$ & $\begin{array}{l}\text { - } \quad \text { Krimer et al. } \\
2016\left(^{*}\right)\end{array}$ \\
\hline $\begin{array}{l}\mathrm{N}- \\
\text { hypervolume } \\
\text { (Blonder et al. } \\
\text { 2014) }\end{array}$ & $\begin{array}{l}\text { Computation of } \\
\text { n-hypervolume }\end{array}$ & $\begin{array}{l}\text { Level of } \\
\text { functional } \\
\text { space filled by } \\
\text { the community }\end{array}$ & $\begin{array}{l}\text { - Taking into } \\
\text { account all } \\
\text { species } \\
\text { Taking into } \\
\text { account } \\
\text { disjunctions and } \\
\text { holes in the } \\
\text { functional space }\end{array}$ & $\begin{array}{l}\text { Subjective choice } \\
\text { of two key } \\
\text { parameters for the } \\
\text { computation } \\
\text { Sensitive to the } \\
\text { dimensionality of } \\
\text { functional space } \\
\text { Sensitive to the } \\
\text { size of dataset }\end{array}$ & $\begin{array}{l}\text { - Lamanna et } \\
\text { al. } 2014\end{array}$ \\
\hline $\begin{array}{l}\text { FRim } \\
\text { (Schleuter et } \\
\text { al. 2010) }\end{array}$ & $\begin{array}{l}\text { Integral of the } \\
\text { maximum of all } \\
\text { fs functions }\end{array}$ & $\begin{array}{l}\text { Occupation and } \\
\text { span of trait } \\
\text { space }\end{array}$ & $\begin{array}{l}\text { - } \quad \text { Taking into } \\
\text { account the ITV }\end{array}$ & $\begin{array}{l}\text { Strongly sensitive } \\
\text { to the size of } \\
\text { dataset } \\
\text { Long computation } \\
\text { time }\end{array}$ & $\begin{array}{l}\text { - Schleuter et } \\
\text { al. } 2012\left({ }^{* *}\right)\end{array}$ \\
\hline $\begin{array}{l}\text { TPD } \\
\text { (Carmona et } \\
\text { al. 2016) }\end{array}$ & $\begin{array}{l}\text { Sum of } \\
\text { hypervolumes } \\
\text { of the cells } \\
\text { where TPD >1 }\end{array}$ & $\begin{array}{l}\text { Amount of } \\
\text { functional } \\
\text { volume } \\
\text { occupied by a } \\
\text { TPD }\end{array}$ & $\begin{array}{l}\text { - } \quad \text { Taking into } \\
\text { account the ITV }\end{array}$ & $\begin{array}{l}\text { - } \quad \text { Need for } \\
\text { extensive } \\
\text { observations per } \\
\text { FU }\end{array}$ & $\begin{array}{l}\text { - No studies } \\
\text { found }\end{array}$ \\
\hline
\end{tabular}




\section{FIGURES}

Figure 1: Schematic representation of the computation of the N-hypervolume index of Blonder et al. (2014). KDE: Kernel Density Estimator.

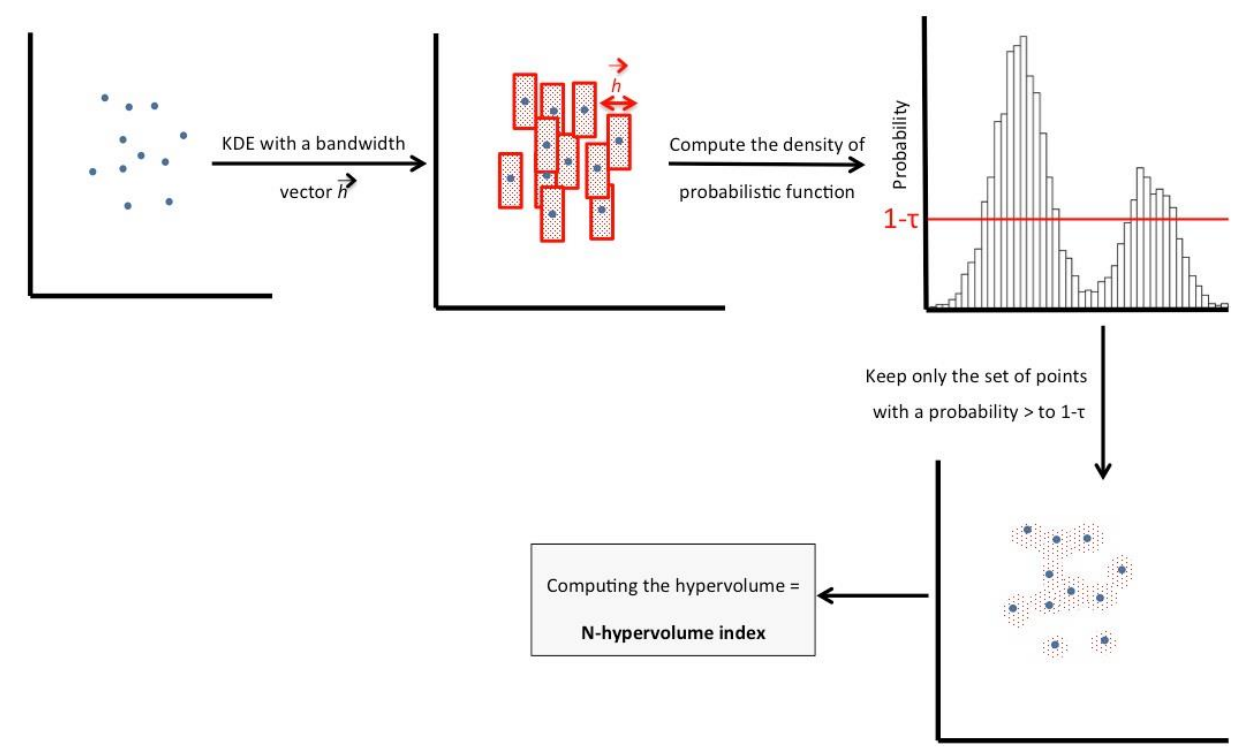

Figure 2: An example of FRim index computation (Schleuter et al. 2010) of three assemblages (for one-dimensional data) characterizing with the species trait values: $A 1=[3.5,4,4.5]$, $\mathrm{A} 2=[3.5,3.75,4,4.25,4.5]$ and $\mathrm{A} 3=[3.5,3.75,3.9,4,4.1,4.25,4.5]$, respectively . The three $f_{s}$ functions are represented using black, blue and red lines respectively, and the FRim index value (computing from the formula $f s(x)=\exp \left[-\frac{1}{2} \frac{\left(x_{j}-\mu\right)^{2}}{\sigma^{2}}\right]$ where $\mu$ is the mean trait value and $\sigma$ is the standard deviation trait value) for these three assemblages corresponds to the area under their respective curves.

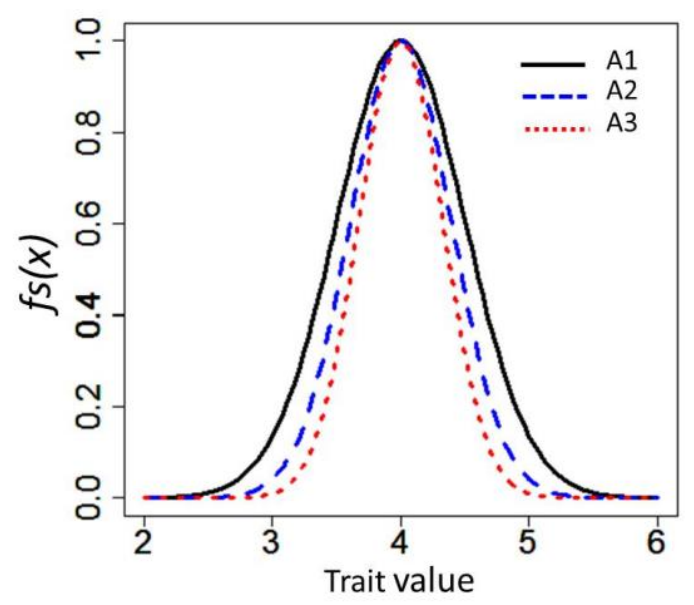


Figure 3: Guidelines for the choice of the functional richness indices by users according to the aims of the study. The grey boxes under each index contain the requisites of the dataset for using the index

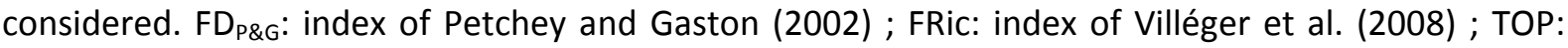
index of Fontana et al. (2015) ; N-Hyp.: index of Blonder et al. (2014) ; FRim : index of Schleuter et al.

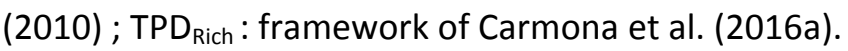

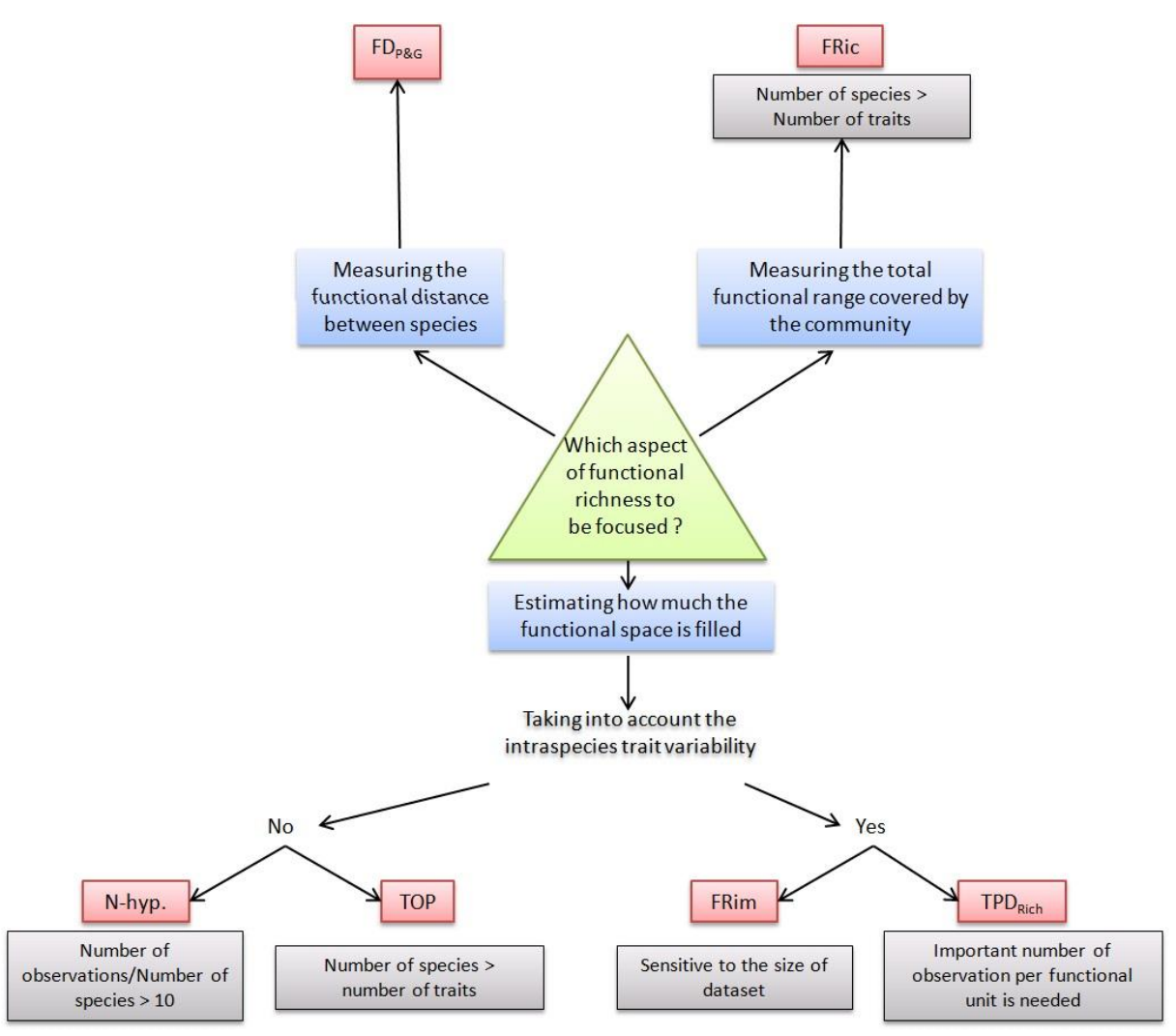


BOX 1: Changes of three multidimensional-based method index values along gradient of different types of disturbances

With the aim of comparing the FRic, TOP and N-hypervolume indices, i.e. the three indices based on the concept of functional space (and on the projection of species trait values in this space), we simulated three scenarios of disturbances resulting in different losses of functional units, and we displayed the responses of these three indices in the face of these disturbances. The three scenarios considered are inspired by those developed by Schleuter et al. 2010, Mouillot et al. 2014 and Loiseau et al. 2017, and they characterize different examples of disturbances affecting communities. We consider four functional traits and an initial species richness of 50 species. Trait values for each species were simulated under a normal distribution (mean of 0 and standard deviation of 1 ). Thus, each species accounts for a unique combination of functional trait values. Scenario 1 mainly affects species located in the center of functional space that can notably be characteristic of a biotic pressure (e.g. introduction of non-native species in the center of the functional space, Mouillot et al. 2014). For that purpose, we deleted at each time step the species that is the closest to the center of gravity of the functional space (as simulated in Schleuter et al. 2010). Scenario 2 simulated a disturbance that impacts species populations with high values for one functional trait, as in Mouillot et al. 2014. Such a situation may notably occur in response to human impacts such as overfishing (Mouillot et al. 2014). For that purpose, we deleted at each time step the species that have the highest values for the first functional trait. Scenario 3 represents a loss of functional units that could occur along an environmental gradient due the differences of niche availability (Loiseau et al. 2017). Here, we deleted at each time step the species located in the extreme parts of the functional space. For simplicity, the illustration of three scenarios is represented in two dimensions. 


\section{Scenario 1}

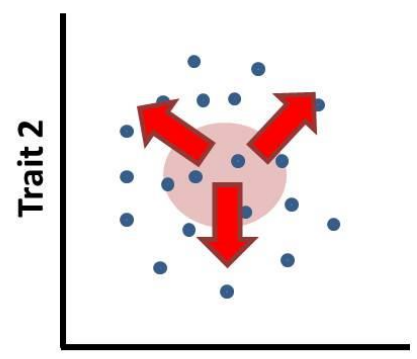

Trait 1
Scenario 2

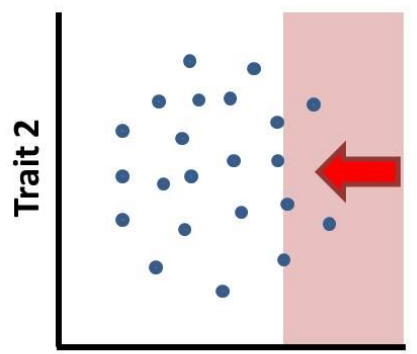

Trait 1
Scenario 3

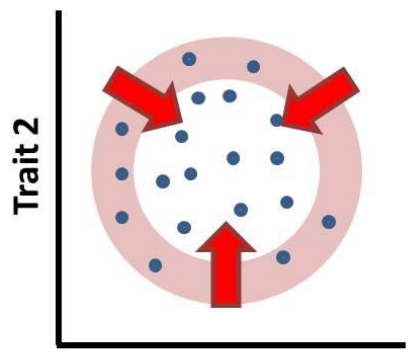

Trait 1

FRic

TOP

N-hyp.
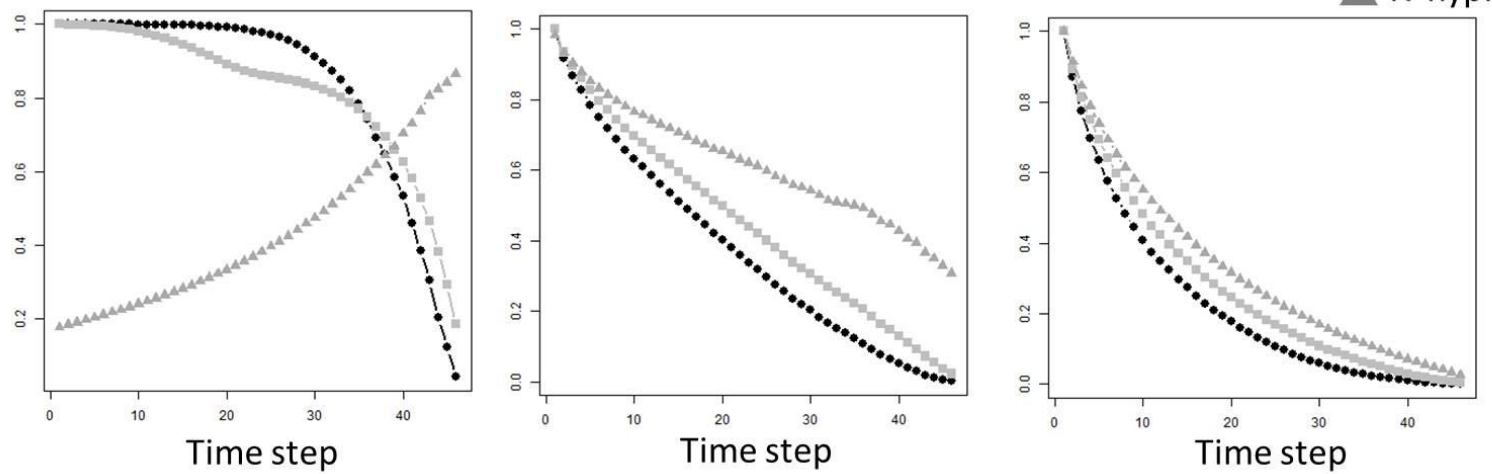

Figure 1: Changes in index values observed during the three scenarios presented above. (a) In scenario 1, the TOP index decreased during the simulation process. The FRic index is weakly impacted up to the middle of the simulation process and strongly decreased after that. The $\mathrm{N}$ hypervolume index (N-hyp.) increases throughout the simulation process (i.e. with the intensification of the disturbance) that is counter-intuitive. (b) In Scenario 2, the three indices gradually decreased over the simulation process, but the decrease of $\mathrm{N}$-hyp. is weaker than those observed for the two other indices (i.e. FRic and TOP indices). (c) In Scenario 3, the three indices decreased during the simulation process in a similar way. Standard errors of mean were computed for each point of three simulations, but these values were too small to be represented or to be visible in the corresponding plots. 